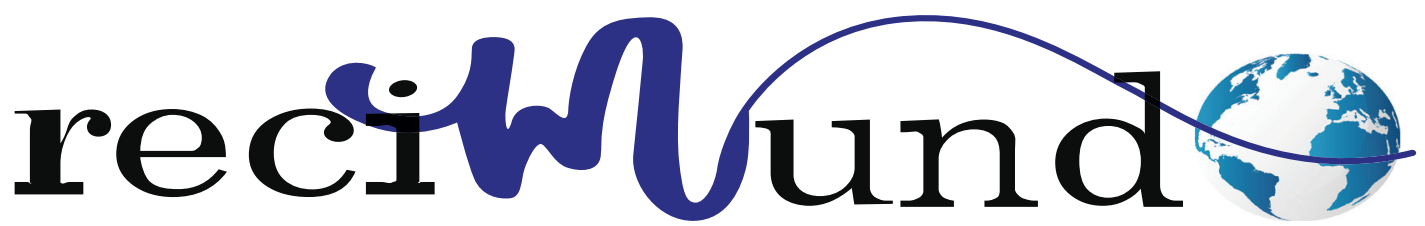

Revista Científica Mundo de la Investigación y el Conocimiento

DOI: 10.26820/recimundo/4.(1).esp.marzo.2020.133-142

URL: http://recimundo.com/index.php/es/article/view/787

EDITORIAL: Saberes del Conocimiento

REVISTA: RECIMUNDO

ISSN: 2588-073X

TIPO DE INVESTIGACIÓN: Artículo de Revisión

CÓDIGO UNESCO: 3205 Medicina Interna

PAGINAS: 133-142

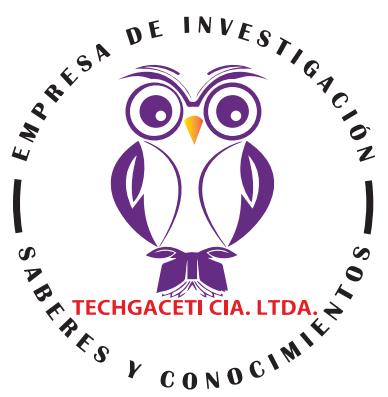

\title{
Manejo de quemaduras eléctricas
}

\section{Management of electrical burns}

Gestão de queimaduras elétricas

\section{Alexandra Estefanía Velasco Cargua'; Ana Karina Díaz Chávez; Jessica Adriana Espín Jiménez; \\ Jhonny Fabián Ruíz Satán ${ }^{4}$}

RECIBIDO: 20/11/2019 ACEPTADO: 29/01/2020 PUBLICADO: 05/03/2020

1. Médico; Investigadora Independiente; Quito, Ecuador; stefy0104alexa@outlook.com; (D) https://orcid.org/00000002-4389-1355

2. Médico; Investigadora Independiente; Quito, Ecuador; akdiazchavez@gmail.com; (D) https://orcid.org/00000003-3328-2377

3. Médico; Investigadora Independiente; Quito, Ecuador; jessadri.1990@hotmail.com; (D) https://orcid.org/00000002-0773-2920

4. Médico General; Investigador Independiente; Riobamba, Ecuador; jhonny_sdb@hotmail.com; (D) https://orcid. org/0000-0002-5143-3741

CORRESPONDENCIA

Alexandra Estefanía Velasco Cargua

stefy0104alexa@outlook.com

Quito, Ecuador

๑ RECIMUNDO; Editorial Saberes del Conocimiento, 2020 


\section{RESUMEN}

La electricidad, es una de las principales fuentes de energía utilizada en el ámbito doméstico e industrial, tal popularidad supone ciertos riesgos asociados a su utilización, así como lo puede ser una quemadura eléctrica. El efecto del contacto entre el cuerpo humano y la energía eléctrica puede ser catastrófico, de acuerdo al tipo y potencia de la corriente eléctrica, el voltaje, resistencia del tejido expuesto y tiempo de exposición, puede ocasionar desde lesiones superficiales, hasta lesiones profundas, afectando negativamente el correcto funcionamiento orgánico. El tratamiento inicial recomendado se basa en fluidoterapia y monitoreo cardíaco, una vez estabilizado el paciente se procederá con las intervenciones quirúrgicas a las que haya lugar, tales como remoción de la quemadura, cobertura de heridas, injertos cutáneos, para finalmente entrar en la fase de cirugía reconstructiva.

Palabras clave: Quemadura eléctrica, Corriente eléctrica continua, Corriente eléctrica alterna, Fluidoterapia, Síndrome compartimental, Injerto cutáneo, Desbridamiento, Tejido necrótico.

\section{ABSTRACT}

Electricity is one of the main sources of energy used in the domestic and industrial field; such popularity involves certain risks associated with its use, such as an electric burn. The effect of the contact between the human body and the electrical energy can be catastrophic, depending on the type and power of the electric current, the voltage, resistance of the exposed tissue and exposure time, can cause from superficial lesions, to deep lesions, affecting negatively the correct organic functioning. The recommended initial treatment is based on fluid therapy and cardiac monitoring, once the patient is stabilized, the surgical procedures will be carried out, such as burn removal, wound cover, skin grafts, to finally enter the phase of reconstructive surgery.

Keywords: Electric burn, Continuous electric current, Alternating electric current, Fluid therapy, Compartment syndrome, Skin graft, Debridement, Necrotic tissue.

\section{RESUMO}

A eletricidade é uma das principais fontes de energia utilizadas no campo doméstico e industrial; essa popularidade envolve certos riscos associados ao seu uso, como uma queimadura elétrica. O efeito do contato entre o corpo humano e a energia elétrica pode ser catastrófico, dependendo do tipo e potência da corrente elétrica, da tensão, da resistência do tecido exposto e do tempo de exposição, que pode causar lesões superficiais a profundas, afetando negativamente o correto funcionamento orgânico. O tratamento inicial recomendado é baseado em fluidoterapia e monitoramento cardíaco. Uma vez estabilizado o paciente, serão realizados procedimentos cirúrgicos, como remoção de queimaduras, cobertura de ferida, enxertos de pele, para finalmente entrar na fase da cirurgia reconstrutiva.

Palavras-chave: Queimadura elétrica, Corrente elétrica contínua, Corrente elétrica alternada, Fluidoterapia, Síndrome do compartimento, Enxerto de pele, Desbridamento, Tecido necrótico. 


\section{Introducción}

La electricidad se ha convertido en un recurso indispensable de la sociedad moderna, su amplia utilización en el ámbito doméstico e industrial ha traído como consecuencia una creciente posibilidad de ocurrencia de accidentes, tal como lo son las quemaduras eléctricas. Las quemaduras eléctricas se consideran un tipo particular de quemadura, debido a su fisiopatología complicada y diversa; su tratamiento debe estar fundamentado en el entendimiento del funcionamiento de la electricidad y su trayectoria dentro del organismo.

El manejo adecuado de una quemadura eléctrica, requiere del conocimiento y comprensión de las propiedades físicas de la electricidad, como lo son el tipo de corriente eléctrica (continua o alterna), el voltaje, la resistencia del tejido y el tiempo de exposición o contacto, ya que éstos son factores determinantes de la severidad de la quemadura o lesión. A través de la presente investigación, se ofrece una detallada explicación de las propiedades físicas de la electricidad y su relación con las quemaduras eléctricas, tipología de quemaduras y el manejo que debe aplicarse ante tal escenario.

\section{Metodología}

La metodología aplicada en la presente investigación radica en una revisión bibliográfica documental y descriptiva, en la que se acudió a diversas publicaciones electrónicas de carácter científico, artículos arbitrados así como publicaciones institucionales, en el ámbito internacional, a fin de presentar un vasto compendio de información referente al tratamiento de las quemaduras eléctricas.

\section{Conceptos básicos de la electricidad}

El concepto de electricidad es definido por diversos autores como el movimiento continuo de electrones que forman los átomos. Los elementos que constituyen la electrici- dad son los siguientes:

- Corriente eléctrica: se genera con el desplazamiento de cargas eléctricas a través de un conductor, de acuerdo al Sistema Internacional de Unidades la intensidad de una corriente eléctrica es medida en amperios cuyo símbolo es A. De acuerdo a la dirección del flujo de electrones, se puede clasificar en:

- Continua o directa (CC): es aquella corriente donde el flujo de electrones va hacia una misma dirección, es decir su sentido de circulación es siempre el mismo, independientemente de su valor absoluto; La corriente eléctrica generada por las baterías, es un ejemplo común de este tipo de energía.

- Alterna (CA): es el tipo de corriente eléctrica en la que los electrones cambian de dirección y magnitud de manera cíclica; es la forma más común de corriente utilizada en hogares y empresas.

- Voltaje: es definido como la energía necesaria para hacer posible el trayecto de una carga entre dos puntos, es medida en voltios cuyo símbolo es $\mathrm{V}$.

- Resistencia: es el obstáculo al flujo de corriente o electrones a través de un conductor, se mide en ohmios y es representado simbólicamente con la letra griega omega $(\Omega)$. El denominado efecto o ley de Joule, es un fenómeno directamente asociado a este elemento de la electricidad, el mismo establece que la resistencia aplicada al flujo eléctrico en un conductor es directamente proporcional a la temperatura generada en dicho conductor, es decir a mayor resistencia mayor será el calor generado.

\section{Las quemaduras eléctricas}

Una quemadura, es definida como "una lesión en tejidos vivos, causada por la acción de diversos agentes, se manifiestan

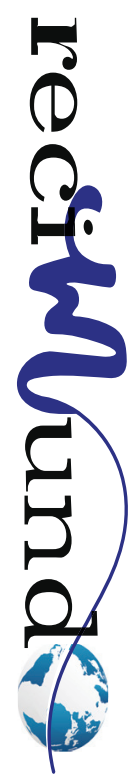


desde un enrojecimiento hasta la destrucción total de las estructuras afectadas" (Paredes). Las quemaduras eléctricas son el resultado de la exposición o contacto con una fuente de corriente eléctrica, son catalogadas (Casteleiro \& Castro Prado) como las más devastadoras de todas las lesiones térmicas, debido al calor de alta intensidad producido por el cuerpo de la víctima cuando se convierte en una resistencia de la corriente eléctrica; afectan normalmente grandes proporciones de piel y tejidos más profundos como: músculos, tendones, huesos, articulaciones, arterias, nervios y otros órganos.

La corriente eléctrica por sí sola no posee temperaturas térmicas elevadas, pero al entrar en contacto con los tejidos del organismo, se generan altas temperaturas producto de la resistencia que dicho tejido supone para la corriente eléctrica. Las quemaduras eléctricas se caracterizan usualmente por ser muy profundas y no exhibir en la superficie corporal o piel, el daño realmente causado, por lo que el porcentaje de superficie corporal visiblemente comprometida no es garantía del deterioro realmente existente, razón por la cual guardan una gran diferencia con las quemaduras térmicas habituales cuya afectación se limita a las áreas y tejidos externos.

Diversos autores coinciden en que las quemaduras o lesiones eléctricas se pueden clasificar en 3 tipos, las mismas se describen a continuación:

Quemadura corriente eléctrica directa: es originada por el trayecto de la corriente eléctrica entre dos puntos orgánicos, de manera tal que se incluye al cuerpo humano en el circuito eléctrico. Este tipo de quemaduras generalmente presentan laceraciones de entrada y salida, y en su recorrido el daño es principalmente térmico; la magnitud, duración y frecuencia de la corriente eléctrica, son determinantes en la extensión y gravedad de la quemadura.
Quemadura por arco eléctrico: son aquellas que se producen al entrar en contacto con el arco producido por dos superficies eléctricamente cargadas y que no están en contacto. La quemadura es producida por las altas temperaturas del arco eléctrico (de $3000^{\circ} \mathrm{C}$ a $10000^{\circ} \mathrm{C}$ ). La corriente eléctrica exógena, entra al cuerpo desde el área de contacto hacia la tierra; la cercanía entre el cuerpo y el arco, así como la temperatura generada por el arco, son factores determinantes de la magnitud y profundidad de la quemadura. Pueden ser producidas por corrientes eléctricas de bajo o alto voltaje, y suelen comprometer una extensión cutánea superior a las quemaduras por corriente eléctrica directa.

Quemadura ignición: Son quemaduras producidas por llamas producto de la incineración generada por la corriente eléctrica en la ropa de la víctima o en algún objeto inflamable cercano.

Propiedades físicas de la electricidad y su relación con las lesiones:

Las propiedades físicas de la electricidad (Corriente eléctrica. Voltaje y Resistencia) son factores determinantes en la generación de lesiones, además de ser elementales para la predicción del estado final del paciente, así como para el tratamiento de dicha lesiones. La gravedad de las quemaduras depende de cuatro propiedades físicas de la fuente eléctrica, éstas son: tipo de corriente, voltaje, resistencia de los tejidos y lapso de exposición.

Tipo de corriente:

- Corriente eléctrica continua o directa (CC): Normalmente produce sólo una contracción muscular que por lo general retira a la víctima del punto de contacto, razón por la cual se le considera como una fuente poco peligrosa.

- Corriente eléctrica alterna (CA): Puede generar de 40 a 110 contracciones por segundo en el sujeto afectado; las conti- 
nuas contracciones generan un fenómeno llamado tetania, que pueden adherir a la víctima a la al sitio de contacto, alargando el tiempo de exposición y maximizando el daño producido. Adicionalmente puede generar una contracción anormal en el tórax dificultado la respiración y arritmias cardíacas.

\section{El voltaje}

- Bajo voltaje: cuando el voltaje de la corriente eléctrica es igual o inferior a 1000V, es el tipo de lesión eléctrica más común, usualmente los afectados son niños y jóvenes en los hogares, normalmente manifestando lesiones de boca y mano por contacto con tomacorrientes. El uso de herramientas eléctricas en mal estado, es una de las principales causas de lesiones en adultos, pudiendo generar contracciones tan fuertes que pueden caudar fracturas o dislocaciones, además de quemaduras en la piel.

- Alto voltaje: corrientes eléctricas con voltajes superiores a $1000 \mathrm{~V}$, usualmente causadas por contacto directo con líneas de alimentación. Puede causar desde lesiones superficiales en la piel, hasta lesiones profundas que comprometen los músculos, pudiendo generar necrosis muscular liberando al torrente sanguíneo creatina fosfoquinas y mioglobina, sustancias que en altas concentraciones pueden acarrear insuficiencia renal aguda.

\section{La resistencia}

La cantidad de agua o cualquier líquido presente en el tejido, en un factor determinante en la resistencia del cuerpo humano ante la electricidad; la humedad del tejido es inversamente proporcional a la resistencia de la misma, es decir que mientras mayor sea la humedad del tejido menor será su resistencia, generando una mayor conducción de corriente eléctrica. Por otro lado, se debe tener en cuenta la ley de Joule, que plantea un aumento de la energía térmica directamente proporcional a la resistencia, es decir que mientras mayor sea la resistencia del tejido, mayor será el calor generado. En la tabla a continuación se detallan los niveles de resistencia (expresados en ohmios) de los órganos y tejidos principalmente afectados por quemaduras eléctricas.

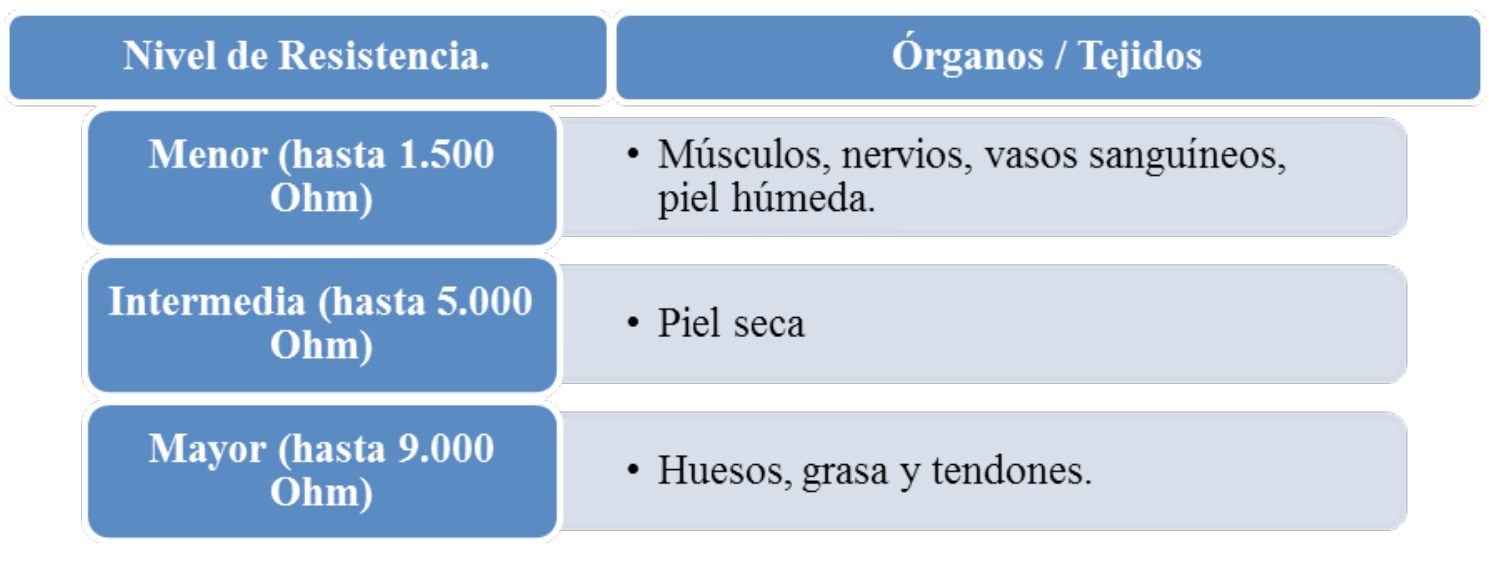

Tabla 1. Resistencia eléctrica de los tejidos humanos

Fuente: (Gonzalez Castro, Ávila Vargas, Quezada Rueda, \& Vivas García, 2018) 


\section{Tiempo de exposición}

El tiempo de contacto con la fuente eléctrica es un factor clave en la determinación del grado de la lesión, así como en la evolución de energía eléctrica a energía térmica, mientras mayor sea el lapso de tiempo en contacto con la corriente eléctrica, mayor será la energía térmica generada, y por ende la quemadura será exponencialmente más grave.

\section{Manejo de las quemaduras eléctricas}

Debido a la fisiopatología tan complicada y diversa que generan las quemaduras con electricidad, su manejo y tratamiento debe ser diferente al aplicado a las quemaduras convencionales con llamas. El manejo de victimas de quemaduras eléctricas, debe ser absolutamente multidisciplinario, deben intervenir especialistas en terapia intensiva, cirugía plástica y reconstructiva, nefrología entre otros.

\section{Manejo inicial}

Un adecuado manejo de las quemaduras eléctricas, debe comenzar en el lugar donde el herido sufre la lesión, para posteriormente ser trasladada a la sala de emergencias, tal como lo plantea (Leyva \& Carvajal Flechas, 2015): "la evaluación inicial debe seguir los protocolos establecidos por el Programa de Soporte Vital Avanzado en Trauma (ATLS, por su sigla en inglés)". Primordialmente se debe catalogar a la víctima como paciente con múltiples traumatismos, ya que gran número de éstos, aunado a la quemadura eléctrica presentan lesiones por caídas desde alturas, o impacto contra objetos, además de fracturas debido a las fuertes contracciones musculares que puede producir la corriente eléctrica. Seguidamente se debe dar prioridad a la evaluación y protección de las vía respiratorias, para continuar con la reanimación y posterior estimación de posibles lesiones traumáticas no térmicas.

\section{Fluidoterapia}

La reposición de fluidos a través de la vía parenteral, el elemento terapéutico más importante del tratamiento general en el quemado grave, ya que puede llegar a extender la supervivencia de las victimas más allá de las 72 horas, superando el shock hipovolémico.

El signo de témpano de hielo o iceberg, que expone una aparentemente pequeña zona 'de piel afectada, bajo la cual se encuentra grandes áreas compromiso tisular, hacen que sea cuesta arriba la predicción de las proporciones de líquido necesario a ser suministrado por vía intravenosa, motivo por el cual no deben utilizarse la formulas tradicionales de cálculo.

Considerando que las quemaduras eléctricas, generan un daño profundo y poco visible en la superficie exterior, no es recomendable la utilización de la fórmula de Parkland (comúnmente utilizada para estimación de infusión en quemados), ya que dicha fórmula al basarse únicamente en el área de superficie corporal quemada, tiende a subestimar las necesidades reales del paciente. En este sentido, (Leyva \& Carvajal Flechas, 2015) plantea que la tasa de infusión inicial para 24 horas, debe ser de $7 \mathrm{~cm} 3 / \mathrm{Kg}$ de peso $x \%$ de superficie corporal quemada, la mitad del estimado debe infundirse en las primeras 8 horas posteriores a la quemadura.

\section{Monitoreo cardiaco}

Gran parte de las víctimas de quemaduras eléctricas, mueren en los minutos posteriores a la ocurrencia del evento, por consecuencia de la fibrilación ventricular. Sin embargo los casos de lesiones cardíacas fulminantes son más predominantes en ensayos con animales que en humanos, en los que se haya áreas necróticas en el miocardio y hemorragias focalizadas, las arritmias (potencialmente fatales) son los fenómenos usualmente detectados en pacientes de este tipo, razón por la cual la evaluación 
cardíaca se convierte en una necesitad básica en el manejo de las víctimas de quemaduras eléctricas.

A pesar de que posterior a la ocurrencia de la quemadura eléctrica, son comunes las patologías cardíacas, se presume que la mayoría de las complicaciones de este tipo ocurren de manera aguda, ya que es muy poco probable que un paciente desarrolle una arritmia letal días después de ocurrido el evento. En este sentido, es recomendable la ejecución de un electrocardiograma como una parte sistemática de la valoración inicial.

De acuerdo a los resultados arrojados por el mencionado electrocardiograma inicial, si el paciente presenta alteraciones tales como arritmias, paro cardíaco o pérdida de conciencia, es recomendable el monitoreo cardíaco continuo. El tiempo de monitoreo debe ser por lo menos de 24 horas desde la admisión del paciente, en los casos en los que no se evidencie ninguna anomalía en el electrocardiograma inicial practicado al momento del ingreso, si el paciente presentó arritmia o algún otro fenómeno, el monitoreo cardíaco debe ser de al menos 24 horas posterior a la resolución del mismo.

\section{Síndrome compartimental}

La acumulación de líquido en la fascia que delimita los compartimientos, aunada al daño muscular, puede incrementar la presión, llegando al punto de afección de la perfusión muscular, llevando irremediablemente a la necrosis muscular, desarrollada en las primeras 48 horas desde la quemadura, por lo que comúnmente el tratamiento del síndrome compartimental, era la aplicación inmediata dentro de las primeras 24 horas, de una exploración y descompresión quirúrgica, seguido de una serie de desbridamientos necesarios para su posterior cierre. Usualmente, las quemaduras por contacto con altos voltajes son relacionadas con daños tisulares más profundos, mucho mayores a los que se visualizan en la superficie de la piel, lo que se traduce en impor- tantes cifras de amputaciones en este tipo de lesión.

Esta impetuosa práctica estaba fundamentada en el principio de Joule (energía térmica es directamente proporcional a la resistencia del tejido), siendo el hueso el tejido con mayor resistencia a la corriente eléctrica, produce importantes temperaturas térmicas provocando la necrosis del tejido muscular cercano. Partiendo de esta premisa, la exploración y descompresión quirúrgica inmediata, supondría la preventiva de pérdidas tisulares asociadas a la hidropesía masiva y el incremento en la presión de los compartimentos, no obstante esta práctica supone la realización de varios procedimientos, lo que prolonga la estancia hospitalaria y aumenta la morbilidad.

En la actualidad, la literatura clínica ha apoyado un manejo mucho más cuidadoso del procedimiento de descompresión quirúrgica inmediata, descartándola del protocolo de atención primaria del paciente con quemaduras eléctricas; lo que se traduce en reducción en la cifra de intervenciones y por consiguiente disminución de la tasa de amputaciones y morbilidad. Las indicaciones para la práctica de una descompresión quirúrgica actualmente son: disfunción nerviosa periférica progresiva, aumento en la presión del compartimiento, mionecrosis inminente y afectación vascular.

\section{Manejo quirúrgico del paciente quemado}

En las primeras 24 a 72 horas de la quemadura, es dificultosa la delimitación entre el tejido necrótico y el viable, razón por la cual el desbridamiento quirúrgico en los casos donde las quemaduras comprometieron la totalidad del espesor de la piel, deben comenzar a ejecutarse tres días después de la quemadura. Se debe realizar el desbridamiento de todo el tejido necrótico identificado, y el tejido parcialmente vital debe ser reevaluado cada dos días hasta completar el cierra completo de la herida.

Se debe ser extremadamente cuidadoso en

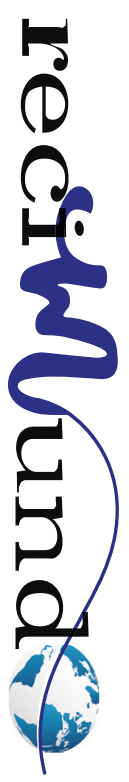


el aseo quirúrgico, retirando la totalidad del tejido necrótico previo al cierre de la herida, a fin de garantizar la salubridad del tejido vital restante, ya que el mínimo residuo de tejido desvitalizado supone un gran riesgo bacteriológico susceptible a complicaciones infecciosas.

La consecución de resultados funcionales óptimos, está condicionada por el desbridamiento conservador ante el agresivo, así como el pronto cierre definitivo de heridas mediante la utilización de injertos de piel o colgajos. La herida debe estar cubierta en todo momento, bien sea por injertos o apósitos que mantenga un ambiente propicio para la correcta cicatrización, lo que se traduce en resultados favorecedores en el cierre definitivo de la herida.

Es de vital importancia la planificación de las intervenciones quirúrgicas, orientadas a la minimización de las mismas, ya que la utilización de anestesia supone un alto riesgo para el paciente. La magnitud en cuanto a tamaño y profundidad de la quemadura serán los determinantes en la elección de la cirugía a practicar. Los procedimientos deben ser abordados de manera multidisciplinaria incluyendo en el equipo de atención terapistas, que estén en facultad de asistir al médico en la toma de decisiones que prioricen la utilización y posicionamiento de colgajos cutáneos. Orientados a la preservación funcional y cosmética de la superficie de la piel.

\section{Remoción de la quemadura}

Es el proceso de eliminación temprana del tejido necrótico de manera quirúrgica bajo los efectos de la anestesia, en aras de resecar el tejido muerto producto de la quemadura, mediante la utilización de un electrocauterio, bisturí o escalpelo. Según (Mariano López, 2003) los mecanismos de remoción de quemaduras utilizados habitualmente son:

- Las escisiones tangenciales: consisten en remover el tejido en capas secuen- ciales con base en una dermis viable y usualmente una escisión de los estratos profundos de la piel, se realizan en quemaduras con profundidad de espesor parcial, después de las 48 horas y dentro de la primera semana posterior a la quemadura, se utiliza un dermátomo de Brown.

- La escisión de espesor total o escarectomía: consiste en remover tejido necrótico hasta encontrar tejido celular subcutáneo o fascia. La remoción se realiza haciendo una incisión en la interfase entre la piel normal y la escara. La incisión se prolonga dentro de tejido viable debajo de la escara hasta removerla en su totalidad.

La exploración quirúrgica de tejido necrótico en la membrana de tejido conjuntivo que recubre el exterior de los huesos, debe hacerse de manera agresiva, sin obviar las quemaduras causadas por arco eléctrico, en la articulaciones y áreas de flexión, donde se concentran mayor cantidad de tendones, tejidos que por su alta resistencia a la corriente eléctrica, presumen mayor daño en las áreas adyacentes a su ubicación.

El sangrado muscular, no es necesariamente factor determinante en la viabilidad del músculo, en ocasiones puede no ser un signo certero para afirmar la viabilidad del mismo, en este sentido, el grado de vitalidad del músculo debe ser determinado únicamente por la respuesta ante el estímulo con electrocauterio

Las principales desventajas presentes en el procedimiento de escarectomía son las de carácter cosmético, ya que por las grandes magnitudes de piel removida queda comprometida gran parte del tejido dérmico superficial. Adicionalmente se tiene el riesgo de presentar edema distal, afectaciones nerviosas. En este sentido queda a discreción del cirujano tratante la elección entre una escisión tangencial o una de espesor total. 


\section{Injertos cutáneos}

Al ser completado en su totalidad el proceso de remoción de la quemadura o escarectomía, el siguiente paso en el manejo quirúrgico de un paciente quemado, es el de cobertura de la herida. Principalmente cuando es precedido por un gran compromiso tisular producto de la escisión de espesor total, o cuando existe una lesión por escisión parcial con una antigüedad de 21 días sin mostrar signo de cicatrización, se hace necesaria la utilización de autoinjertos, es decir un trasplante de piel desde un punto donador hasta el punto receptor. Los injertos de piel son adheridos con grapas o suturas al sitio que el cirujano considere pertinente, para posteriormente ser cubiertos con apósitos o vendajes de acuerdo a indicaciones del cirujano.

Seguidamente el cirujano tratante, debe realizar una planificación de posicionamiento postoperatorio para el paciente, a fin de garantizar la inmovilización y elongación del injerto, hasta que se normalice la circulación sanguínea o revascularización en la pieza injertada. El dolor del paciente se verá aliviado en la medida en la que la herida sane, siendo de vital importancia el apoyo nutricional y la terapia de rehabilitación física.

\section{Fase reconstructiva}

Una vez superada las fases previas de remoción de quemaduras e injerto cutáneo, se puede estudiar la posibilidad de aplicación de una gran variedad de procedimientos quirúrgicos reconstructivos. Dependiendo de la región afectada y la magnitud del tejido comprometido, así como la experiencia del cirujano se podrán elegir las diferentes alternativas disponibles.

A continuación se listan algunas de las aplicadas usualmente:

- Z-plastias.

- W-plastias.
- V-Y-plastias.

- Utilización de expansores tisulares.

- Cirugía con colgajos.

\section{Conclusiones}

El contacto del cuerpo humano con la energía eléctrica, puede generar daño comprometedor en cualquier órgano o tejido, como piel, músculos, vasos, nervios, tendones hasta huesos; estos daños pueden evolucionar y convertirse en daño cardíaco, renal o neurológico. Las consecuencias físicas y psicológicas pueden ser aciagas para las víctimas, reflejándose en afectaciones negativas en el entorno social y laboral.

El manejo clínico y quirúrgico del paciente con quemaduras eléctricas, es un factor esencial para contrarrestar los posibles daños y complicaciones que una quemadura eléctrica puede acarrear.

El manejo clínico debe centrarse en la reposición de fluidos por vía parenteral, tomando en cuenta las fórmulas adecuadas, ya que la severidad de la quemadura visible en la piel, no es de fiar para determinar el grado afección de la lesión interna. Adicionalmente hay que prestar especial atención al funcionamiento orgánico renal, a fin de prevenir en la medida de lo posible una eventual insuficiencia renal aguda producto de la contaminación sanguínea provocada por la necrosis tisular.

El manejo quirúrgico del paciente debe abordarse se manera multidisciplinaria, lo que permitirá la resolución de los problemas multisistémicos que los pacientes con quemaduras eléctricas presentan.

La prevención de este tipo de incidentes, es por mucho la mejor alternativa ante los resultados, por buenos que sean, de cualquier tratamiento de los presentados en la presente investigación. 


\section{Bibliografía}

Bautista, E., Álvarez, M., Vizcarrondo, J., Muñóz, J. L., \& Márquez , E. (2004). Manejo de las quemaduras eléctricas en el Hospital General de Lídice "Dr. Jesús Yerena". Cirugía plástica reconstructiva venezuela, 6(1-2), 26-28.

Casteleiro, R., \& Castro Prado, J. (s.f.). Guía práctica de lesiones por quemadura. Guía n 5. Recuperado el 02 de 2020, de Programa Úlceras Fora > Colección de guías prácticas de heridas del Servicio Gallego de Salud > Guía práctica de lesiones por quemadura. Guía n 5: https://ulcerasfora.sergas.gal/Informacion/Queimaduras-electricas?idio$\mathrm{ma}=\mathrm{es} \&$ print $=1$

Gonzalez Castro, L. F., Ávila Vargas, S. V., Quezada Rueda, J. T., \& Vivas García, S. M. (2018). Fisiopatología de las quemaduras eléctricas: artículo de revisión. REVISTA ARGENTINA DE CIRUGÍA PLÁSTICA, 24(2), 51-56.

La Torre Tang, W. (2003). Quemaduras Eléctricas: Estudio Clínico-Epidemiológico en el Hospital Nacional Guillermo Almenara Irigoyen. Lima Perú.

Leyva, J., \& Carvajal Flechas, F. (2015). Lesiones eléctricas. Universitas Médica, 56(1), 63-74.

Macías Pérez, J. (2018). Quemaduras eléctricas y anestesia. Revista mexicana de anestesiología, 41(1), 109-112.
Mariano López, D. d. (2003). Manejo del paciente con quemaduras por corriente eléctrica. Revista de Sanidad Militar, 57(3), 149-154.

Ministerio de Salud de Chile. (Julio de 2007). Guía Clínica Gran Quemado. Chile.

Paredes, S. (s.f.). COLEGIO DE MÉDICOS DE LA PROVINCIA DE SALTA. Recuperado el 02 de 2020, de http://www.colmedsa.com.ar/files/Quemaduras.pdf

Reynoso Campo, R., \& Valero Ontiveros, U. J. (2013). Protocolo de reconstrucción microquirúrgica y salvamento de extremidades en quemaduras eléctricas de alto voltaje. Revista Brasileira de Queimaduras, 12(4), 1-7.

Rodríguez Salazar, O., \& Fuentes Díaz, Z. (2011). Protocolización de la atención al paciente con quemaduras eléctricas en las primeras 24 horas. Revista Archivo Médico de Camagüey, 15(3), 477486.

Valdés Mesa, S., Borges Muiño, H., \& Palacios Alfonso, I. (2007). Caracterización clínica del paciente quemado por electricidad. Revista Cubana de Medicina Militar, 36(1).

Valencia Hitte, R., \& García Hernández, J. (2009). Quemaduras eléctricas en boca. Perinatología y reproducción humana, 23(2), 116-123.

\section{CITAR ESTE ARTICULO:}

Velasco Cargua, A., Díaz Chávez, A., Espín Jiménez, J., \& Ruíz Satán, J. (2020). Manejo de quemaduras eléctricas. RECIMUNDO, 4(1(Esp)), 133-142. doi:10.26820/recimundo/4.(1).esp.marzo.2020.133-142 